\title{
A UTILIZAÇÃO DA REALIDADE VIRTUAL EM ANÁLISES ERGONÔMICAS E DE USABILIDADE DE EMBALAGENS - uma revisão de literatura
}

\author{
Angélica de S. G. Acioly. \\ DDesign UFPB/PPGDesign UFPE \\ angelica@ccae.ufpb.br \\ Phillipe Burgos \\ Graduando em Design/UFPE \\ phillipeb2@gmail.com \\ Marcelo M. Soares \\ DDesign/PPGDesign UFPE \\ soaresmm@gmail.com \\ Fábio Campos \\ DDesign/PPGDesign UFPE \\ fc2005@gmail.com
}

\begin{abstract}
Resumo: O uso da Realidade Virtual (RV) e da Realidade Aumentada (RA) tem se mostrado cada vez mais possível, graças à redução dos custos de implementação, à disseminação do conhecimento das tecnologias e ao aumento significativo de possibilidades de uso. Assim, mais pessoas, empresas e instituições de ensino e pesquisa têm utilizado a RVA em diversas áreas. Com isso, há uma crescente necessidade de investigar a interação das pessoas com os sistemas de RVA em suas diferentes aplicações. A fim de conhecer o potencial dessas tecnologias para a avaliação de produtos, este artigo apresenta um levantamento de pesquisas publicadas em periódicos científicos internacionais (Journals) sobre o uso da Realidade Virtual (RV) em análises ergonômicas e de usabilidade de embalagens de consumo. Foi utilizado como principal meio de busca o portal de periódicos da CAPES, além dos sites de cada journal.
\end{abstract}

Palavras-chave: Realidade virtual, ergonomia, usabilidade, embalagens.

Abstract: The use of Virtual Reality (VR) and Augmented Reality (AR) has proved increasingly possible thanks to the reduction of implementation costs, the dissemination of knowledge of the technologies and the significant increase of use possibilities. More people, companies, educational and research institutions have used the RVA in different areas. Thus, there is a growing need to investigate the interaction of people with 
RVA systems in its different applications. In order to know the potential of these technologies for the evaluation of products, this article presents a survey of published scientific research in international journals (Journals) of the use of Virtual Reality (VR) in ergonomic and usability analyzes of consumer packaging. The journal portal of CAPES was used as the primary mean of search, in addition of each journal sites.

Keywords: virtual reality, ergonomics, usability, packaging.

\section{INTRODUÇÃO}

Em termos gerais, os sistemas de Realidade Virtual (RV) permitem criar ciberespaços onde através dos quais é possível interagir com objetos e com pessoas a um nível virtual. Nesses espaços, as leis convencionais de espaço e tempo não necessitam ser seguidas com rigor, tudo pode ser simulado desde que possa ser programado. (VINCE apud SILVA et al, 2009)

Segundo Rebelo et al (2011), as abordagens da RV podem se referir a diferentes contextos (militar, saúde, transportes, indústria, entretenimento, patrimônio cultural) ou tipos de aplicações (por exemplo, protótipos virtuais, formação, investigação, teleoperação). De igual forma a Realidade Aumentada (RA), com destaque para o uso atual para ações de marketing e entretenimento.

A utilização da RV e da RA na Ergonomia também tem acompanhado tal avanço, objetivando desde a qualidade de vida do trabalhador, à qualidade dos produtos, ambientes, sistemas, treinamentos e educação (GRAVE et al, 2001).

Em relação ao desenvolvimento de produtos, as ferramentas disponíveis pela RV/RA para análise ergonômica e/ou de usabilidade têm permitido diversos tipos de simulações em diferentes interfaces entre produto e usuário, seja visual, tátil e/ou auditiva. As análises podem ser realizadas em diversas etapas projetuais como: análise de usabilidade, testes de interação do produto com o ambiente, criação de protótipos virtuais a fim de aperfeiçoar a concepção de produtos em seus diferentes aspectos (estéticos, construtivos, ergonômicos, dentre outros), racionalizando tempo e recursos financeiros no desenvolvimento de produtos, dentre outras vantagens.

A RV apresenta ainda, a possibilidade de conciliar as vantagens de estudos de campo e de laboratório, pois permite que o usuário interaja com o produto em um contexto semelhante à situação real, fazendo com que condições de segurança e outras variáveis possam ser controladas. (REBELO et al, 2011)

Há, portanto, uma crescente necessidade de investigação sobre a aplicabilidade e adaptação dos métodos e ferramentas de avaliação emergentes aos diferentes segmentos de produtos e seus usuários.

Para tanto, este estudo está relacionado ao uso de ferramentas de RV em análises de embalagens de consumo (tipo de embalagem direcionadas ao consumidor final, diferenciando-se da embalagem industrial, com ênfase na logística). Produto este, presente na vida das pessoas de diversas faixas etárias, sexos, classes sociais e em inúmeras situações desde muito cedo, o qual apresenta uma necessidade constante de estudos cada vez mais aprofundados. 
As embalagens, comumente, apresentam problemas de interface com o usuário, tanto nos aspectos físicos (de manuseio) quanto de compreensão de informações visuais. O grande desafio nessa relação de interação entre usuário e embalagem, é, sobretudo, eliminar ou minimizar as dificuldades existentes nas interfaces - problemas de usabilidade e de riscos de acidentes. Tanto nos aspectos projetuais dos produtos, como também no processo de informação ao usuário. Principalmente, pela segurança dos usuários. Uma falha no desenho de uma embalagem ou a não compreensão de um aviso podem ter consequências fatais para os consumidores, como no caso de uma embalagem de medicamentos.

São diversas as dificuldade enfrentadas por seus usuários, de diferentes formas e graus, que vão desde a não compreensão de um rótulo/forma/dispositivos de abertura/fechamento até a ocorrência de desconfortos e/ou acidentes quanto à sua manipulação. Resultando, segundo PRO TESTE e AMB (2005), em diversos acidentes e/ou dificuldades como: cortes, perfurações, ausência de informação sobre o uso ou sobre como descartar a embalagem, explosão, abertura inadequada ou sem trava em produto perigoso. Tais dificuldades de uso e as suas conseqüências podem ser evidenciadas em segmentos de populações como crianças, idosos, pessoas com mobilidade reduzida nos membros superiores e pessoas canhotas.

Zunjic (2011) apresenta como uma das causas da recorrência dos problemas enfrentados na incompatibilidade ergonômica das embalagens, o fato que os fabricantes não são muitas vezes conscientes das dimensões dos problemas que os consumidores têm durante a interação com a embalagem. No entanto, mesmo quando eles estão cientes do problema, muitas vezes não sabem como resolvê-los, pois as questões ligadas à usabilidade em embalagens não são simples de serem avaliadas e materializadas, principalmente pela heterogeneidade dos seus consumidores.

A embalagem, portanto é um sistema amplo no que refere aos aspectos de investigação e atuação - construtivos, marketing, logístico, proteção, de interação, dentre outros. Contudo, o aspecto relacionado à interação com o usuário, tanto física como cognitiva, apresenta um dos principais desafios de análise e pesquisas aplicadas, principalmente pela diversidade de tipos e de características dos usuários potenciais.

Assim, buscando conhecer o potencial e relações de uso de ferramentas da RV para análises de embalagens, este artigo tem como objetivo apresentar um levantamento de pesquisas publicadas em artigos de periódicos internacionais (Journals) sobre o uso da Realidade Virtual em análises ergonômicas e de usabilidade de embalagens de consumo.

Em termos gerais, espera-se que os resultados aqui apresentados possam contribuir para a identificação: dos centros de pesquisa na área; do estado da arte das pesquisas desenvolvidas até o momento; das perspectivas de uso da RV e das demandas para futuras pesquisas. A segunda parte desta pesquisa será realizada posteriormente com o levantamento de trabalhos relacionados à RA. O levantamento aqui apresentado coletou dados para a composição do estado da arte de uma tese em Design e Ergonomia que trata da utilização de ferramentas da Realidade Virtual e Realidade Aumentada na avaliação e orientação de uso em embalagens de consumo. 


\title{
2. DESENVOLVIMENTO
}

\subsection{Realidade Virtual}

Tori e Kirner (2006, p.7) definem Realidade Virtual como uma "interface avançada para aplicações computacionais, que permite ao usuário a movimentação (navegação) e interação em tempo real, em um ambiente tridimensional, podendo fazer uso de dispositivos multisensoriais, para atuação ou feedback".

Segundo Kirner e Kirner (2011, p.11) a

\begin{abstract}
realidade virtual, realidade aumentada e suas variações representam técnicas de interface computacional que levam em conta o espaço tridimensional. Nesse espaço, o usuário atua de forma multisensorial, explorando aspectos deste espaço por meio da visão, audição e tato. Conforme a tecnologia disponível, é possível também explorar o olfato e o paladar. Percepções corpóreas, como frio, calor e pressão, estão incluídas no tato, através da pele. Antes do surgimento da realidade virtual e aumentada, as interfaces computacionais se restringiam ao espaço bidimensional da tela do monitor, viabilizando aplicações multimídia com textos, imagens, sons, vídeos e animações.
\end{abstract}

Para compreender a RV e seus desdobramentos, Paul Milgram apresenta a realidade virtual e realidade aumentada como partes de uma realidade mais ampla Virtuality Continuum (virtualidade contínua) denominada também como Mixed Reality (Realidade Misturada). Este conceito da Ciência da Computação estabelece que existe uma escala contínua entre o completamente virtual - realidade virtual, e o completamente real - a realidade. O continuum realidade-virtualidade envolve todas as possíveis variações e composições de objetos reais e virtuais. A figura 1 apresenta graficamente este conceito, onde cada extremo representa os ambientes - real e virtual e no intervalo estão a realidade aumentada (RA) e a virtualidade aumentada (VA). Sendo predominante na RA a inserção de elementos virtuais no ambiente real e na VA com a incorporação de elementos reais ao ambiente virtual. (ZORZAL et al, 2014) Na perspectiva da Virtuality Continuum, Kirner (2008) apresenta a evolução da RV (Figura 2) no contexto tecnológico, desde a década de 1990.

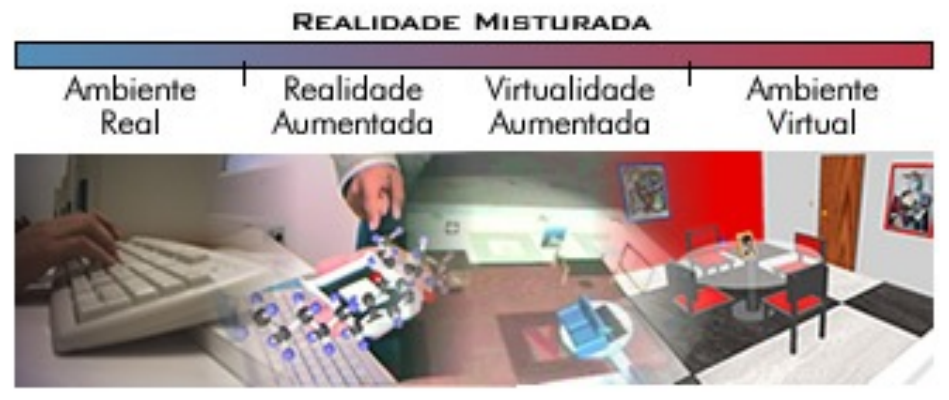

Figura 1. Diagrama de Realidade/Virtualidade contínua Fonte: Milgran apud Zorzal et al (2014)

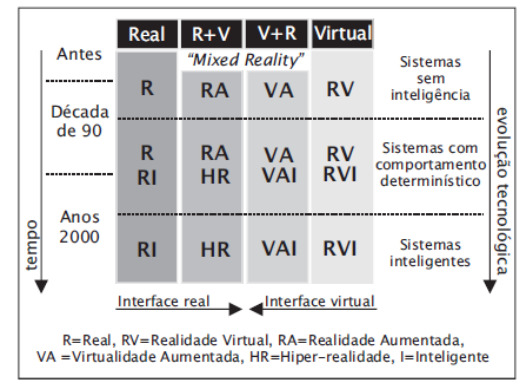

Figura 2 - Evolução da RV . Fonte: Kirner (2008)

Em termos gerais, os sistemas de realidade virtual permitem criar ciberespaços onde através dos quais é possível interagir com objetos e com pessoas a um nível virtual. Nesses espaços, as leis convencionais de espaço e tempo não necessitam ser 
seguidas com rigor, tudo pode ser simulado desde que possa ser programado. (VINCE apud SILVA et al, 2010).

Em termos gerais, a RV é geralmente usada para descrever uma família de tecnologias que apresentem um ambiente virtual ao participante, através de alguns dispositivos de visualização: capacete ou Head Mounted Display ótico ou de vídeo, óculos estereoscópico ou stereo glasses e monitores; dispositivos auditivos: fones de ouvido e microfones externos; e dispositivos táteis e de força: luvas com dispositivos que possibilitam a interação com ações de tato e de força. (WILSON, 1999; AKAGUI e KIRNER, 2004)

\subsection{Procedimentos Metodológicos}

Esta pesquisa pode ser caracterizada como de natureza bibliográfica, e foi estruturada da seguinte forma:

- Levantamento de artigos relacionados à análise ergonômica e de usabilidade de embalagens e Realidade Virtual em 11 periódicos internacionais ligados à ergonomia, design e áreas afins - Applied Ergonomics (ISSN 0003-6870), Ergonomics [(0014-0139 (Impressa), 1366-5847 (Online)], International Journal of Industrial Ergonomics (0169-8141), Human Factors (0018-7208), Design Studies (0142-694x), Computer-Aided Design (0010-4485), leee Software (0740-7459), Computers \& Graphics (0097-8493), Engineering Design 0954-4828 (Print), 1466-1837 (Online) E Packaging Technology and Science (1099-1522). Foi utilizado como principal meio de busca o portal de periódicos da CAPES, além dos sites de cada journal. Foram pesquisados prioritariamente artigos publicados entre os anos de 2008 e 2012. Adicionalmente teve-se o acesso à edição impressa do journal Ergonomics, no período de 2009 a 2013. Contudo, foram selecionados 02 artigos anteriores a esse período, pelo seu caráter histórico. Dos 11 journals pesquisados, foram selecionados artigos em 4 deles. Complementarmente foram levantados artigos também nos Anais do Anais HCI 2013 (ISSN 0302-9743/e-ISSN 1611-3349 / ISBN 978-3-642-39237-5/ e-ISBN 978-3642-39238-2 /DOI 10.1007/978-3-642-39238-2

- Descrição dos artigos selecionados, onde os dados de cada artigo foram sistematizados e apresentando a partir das seguintes informações: título, autores, journal, objetivo(s), síntese dos métodos e ferramentas utilizados e principais resultados.

\subsection{Levantamento}

O principal objeto de estudo desta pesquisa foi a interação do usuário com embalagens com foco nas questões de usabilidade e o uso da realidade virtual como ferramenta pra análise. Desta forma, foram utilizadas como palavra-chave para a identificação dos artigos: análise/avaliação ergonômica e/ou usabilidade de embalagens e/ou produtos de consumo; o uso da realidade virtual na análise de produtos e/ou embalagens.

A partir do levantamento realizado não foram encontrados artigos de RV na avaliação de embalagens de consumo. Desta forma, optou-se em analisar alguns artigos encontrados ligados à: avaliação ergonômica e de usabilidade de produtos de consumo e RV. Nestes termos, foram selecionados quatro artigos de quatro journals 
dos 11 pesquisados. Complementarmente foram selecionados também, dois artigos da 2nd International Conference on Design, User Experience and Usability do $\mathrm{HCl} 2013$, por apresentarem discussões e experimentos com produtos de consumo e RV. Dentre os quais, um com embalagens de consumo.

A sistematização dos artigos selecionados preconizou os seguintes pontos, conforme já descritos: título, autores, journal (ano, edição, volume, páginas), objetivo(s) da pesquisa, materiais e métodos adotados e principais resultados das análises realizadas. Segue a descrição e análise dos artigos selecionados:

\section{- Artigo 1 -Virtual environments applications and applied ergonomics}

Autor: John R. Wilson (Virtual Reality Applications Research Team, Department of Manufacturing Engineering and Operations Management, University of Nottingham, Reino Unido).

Journal: Applied Ergonomics, 1999, v. 30, p. 3-9.

Objetivo(s): Descrever dois casos particulares de desenvolvimento de ambiente virtual relevantes à ergonomia. Este artigo faz parte de uma série de contribuições para uma edição especial sobre ergonomia no estudo e uso de ambientes virtuais.

O autor inicia o artigo discutindo as razões pelas quais os ambientes virtuais (AV) precisaram ser adaptados, na década de 1990, para o uso na indústria, comércio, medicina. E apesar do seu uso se aplicar à diversas situações os estudos aplicados de ergonomia e usabilidade ainda haviam limitações técnicas para tal. Em seguida conceitua Realidade virtual (RV), como se processa a interação entre os elementos envolvidos e tece considerações sobre o ambiente e a tecnologia. Ele aponta um erro comum de compreensão sobre o que é a RV e o que são alguns sistemas e modelos de computadores como os sistemas multimídias, modelos 3D ou sites da WEB. Algumas razões pelas quais a RV é relevante para a aplicação em Ergonomia são apontadas: no projeto de sistemas de RV onde a interação entre os sujeitos da pesquisa e os componentes técnicos de RV devem ser adequados e que promovam conforto físico e psicológico aos participantes; especificação do AV e desenvolvimento e análise de tarefas, onde caberá ao ergonomista entender quais as tarefas devem ser realizadas no domínio em que os sistemas do AV irão ser utilizados e que requisitos e condições irão impor às pessoas envolvidas; usabilidade e interação com o AV onde os ergonomistas podem ajudar a entender como as pessoas vão interagir com o AV; Desenvolvimento e avaliação de aplicações práticas onde deverão ser estudadas como a RV pode ser integrada à outras tecnologias, ou em ambientes mistos ou híbridos; e desenvolvimento e avaliação de ferramentas de ergonomia. A partir do objetivo deste trabalho o autor segue descrevendo duas experiências de uso da RV aplicada a estudos em ergonomia, juntamente com uma avaliação de sua disponibilidade para uso regular. As experiências apresentadas foram: 1. Desenvolvimento e avaliação de AV para treinamento; e 2. Redesenho de trabalho participativo usando AV. Nos dois casos os métodos e técnicas da pesquisa foram apresentados. Os sujeitos da pesquisa participaram de experiências a partir de métodos usuais (físicos) e métodos utilizando AV. Os resultados foram brevemente descritos. Em síntese, nas duas atividades os participantes foram julgados mais capazes e motivados para contribuir no processo de avaliação utilizando o AV. Os resultados se apresentaram satisfatórios no que referem aos métodos que utilizaram a RV.

\section{- Artigo 2 -Virtual reality in the product development process}

Autor: Stig Ottosson (Linköping University, Norrköping, Suécia). 
Journal: Engineering Design, 2002, v. 13, n. 2, p. 159 -172.

Objetivo(s): Apresentar regras para o uso de realidade virtual (RV) no processo de desenvolvimento do produto. Trata principalmente como a RV pode ser utilizada no desenvolvimento do produto, quando deve ser utilizada, e de como ela interage com outro projeto e ferramentas de engenharia.

O artigo inicia com a apresentação de conceitos de realidade virtual e sua utilização em algumas áreas como: aplicações militares, na indústria aeroespacial, entretenimento, automotiva e em áreas de conhecimento como arquitetura, engenharia e design. $O$ autor faz uma prospecção para o futuro do seu uso no mercado de massa, do entretenimento, cirurgia virtual dentre outros, afirmando que no próximo século (neste século) a realidade virtual transformará nossas vidas. Disserta brevemente a respeito de novas formas/ferramentas de desenvolvimento de um produto, tecendo algumas considerações sobre diferentes métodos, tecnologias utilizadas, dinamicidade, rapidez, respostas,... Quando o mesmo se refere no artigo a "produto" ele o relaciona a bens e serviços. O autor considera o uso das ferramentas de RV no desenvolvimento de produtos, apesar de ser usada há certo tempo, como nova na engenharia. Apresenta como vantagem do seu uso: diversas possibilidades para encurtar o tempo de desenvolvimento, para reduzir custos e melhorar a usabilidade e qualidade dos produtos. O autor ressalta que, como o processo de desenvolvimento de produto não é uma ciência exata, suas colocações são opiniões sobre o que ele pesquisou a respeito de tais processos. Em sua investigação apresenta inicialmente um histórico sobre sua experiência pessoal com a RV, bem como as pesquisas que foi desenvolvendo desde 1997. O autor caracteriza suas pesquisas como pesquisa-ação, onde, para melhorar a situação e o ambiente investigados, os diversos profissionais da empresa, dos gestores aos cargos técnicos e também os clientes eram envolvidos. O autor lista algumas de suas pesquisas em empresas como: a adoção e mockups virtuais na Volvo Automóveis; uso da realidade virtual para testar a montagem e desmontagem de um modelo de carro na BMW; uso da realidade virtual pra elaborar demandas e listas de solicitações para os produtos antes da sua prototipagem física na Tetra Pak; Na SCHLUMBERG a utilização da realidade virtual na análise de máquinas. $\mathrm{O}$ autor discute no decorrer do artigo a relação de custo no uso da RV como vantajosa em relação à adoção de protótipos e ambientes físicos e em relação à redução do tempo, contudo apresenta que, algumas ferramentas de análise ainda precisam ser aperfeiçoadas e melhor compreendidas, por exemplo: a luva com sensores que apresenta problemas relativos a sensações realistas. $O$ autor ainda indica que a RV pode também ser usada como método complementar de análise, como suporte de vendas, em feiras por exemplo. Pode contribuir ainda para simulações diversas, inclusive nas que oferecem riscos as pessoas envolvidas, servindo também para treinar habilidades, e por fim, para avaliar aspectos estéticos e ergonômicos de um produto. Em contrapartida, para determinados produtos, pode ser um processo muito caro para algumas empresas. Mas em termos gerais, oferecem uma nova possibilidade para muitas empresas melhorarem a usabilidade de seus produtos com foco em design centrado no usuário. É de forma otimista que o autor finaliza o artigo colocando que o uso da RV lhe parece favorável para o desenvolvimento de produtos, desde que sejam considerados aspectos técnicos, de tempo e custo que essa tecnologia demanda. O autor apresenta o estabelecimento de parcerias com universidades que já montaram centro de pesquisa na área, como uma possibilidade de acesso das pequenas e médias empresas a essa tecnologia. 


\section{- Artigo 3 - Haptic technologies for the conceptual and validation phases of product design}

Autores: Monica Bordegoni, Giorgio Colombo, Luca Formentini (Dipartimento di Meccanica, Politecnico, Itália).

Journal: Computers \& Graphics, 2006, 30, p. 377-390.

Objetivo(s): Apresentar duas aplicações de tecnologias táteis para demonstrar como elas podem aumentar a interação homem-computador durante diferentes etapas do processo de design.

Os autores iniciam o artigo apresentando como a prototipagem virtual tem sido utilizada em vários setores industriais e suas vantagens em relação aos protótipos físicos. Afirmam que a Realidade virtual (RV) hoje tem seu foco no conceito final e nas fases de engenharia e análise e desenvolvimento do produto, e apresentam como tendência recente, a sua utilização para as fases anteriores a essas, tais como avaliação dos conceitos. Logo em seguida, tratam de forma mais detalhada da RV, seu processo evolutivo e os dispositivos interativos existentes, com foco nas tecnologias hápticas (TH) e suas possibilidades de uso. Em síntese, a TH existente permite combinar as capacidades de sistemas de computador com as habilidades tradicionais e métodos de trabalho. $\mathrm{O}$ artigo faz uma ponte entre a modelagem física e a virtual através do uso das tecnologias hápticas. As TH podem desempenhar um papel importante na Prototipagem virtual no que diz respeito à validação dos aspectos ergonômicos. Sobre o objetivo do artigo, ele descreve resultados dos seguintes projetos de pesquisa: 1. TnDTouchand Design - visa o desenvolvimento de um sistema que permita a geração de formas digitais de um forma natural e intuitiva para os modeladores; 2. VERVE2 sistema de RV para validação de controles de equipamentos, projeto centrado na utilização de interfaces hápticas para avaliação ergonômica. $O$ primeiro projeto desenvolveu um sistema de modelagem de produtos industriais baseados em TH. Os requisitos do sistema foram definidos mediante a observação dos designers durante suas atividades diárias de trabalho, no que refere à criação de modelos físicos. As ações de confeç̧ão dos modelos dos objetos selecionados foram filmadas, e realizadas entrevistas com os modeladores. Os dados foram tratados, quanti e qualitativamente, a fim de compreender as vantagens de operar manualmente na criação das formas e suas habilidades manuais, através da identificação e classificação das ferramentas utilizadas e dos gestos e movimentos manuais realizados. Logo após as imagens foram reproduzidas no sistema projetado. O artigo apresenta a arquitetura do sistema projetado para a análise, os métodos de modelagem e as ferramentas táteis adotadas. Por fim, os autores apresentam como o protótipo do sistema foi finalizado e testado. Cerca de 10 designers e engenheiros de CAD foram convidados para o teste, e a partir das suas opiniões, foram feitas as considerações sobre o sistema projetado. No segundo projeto buscou-se avaliar aspectos ergonômicos de painéis de controle compostos por botões utilizados em produtos como carros e eletrodomésticos. 0 primeiro ponto analisado foi o dimensionamento entre os botões e também o posicionamento dos controles. Posteriormente a arquitetura do sistema projetado foi apresentada. Foram desenvolvidos alguns protótipos virtuais de estação de trabalho para os testes com os produtos selecionados, e logo após foi descrito como o dispositivo háptico utilizado para a simulação dos controles foi desenvolvido. Em seguida, o sistema foi testado com os utilizadores. 
- Artigo 4 - Performing ergonomics analyses through virtual interactive design: validity and reliability assessment.

Autores: TinghaoWu ${ }^{1}$, Renran Tian ${ }^{2}$ e Vincent G. Duffy ${ }^{2,3}\left({ }^{1}\right.$ Center for Advanced Vehicle Systems, Mississippi State University, Starkville, MS; ${ }^{2}$ School of Industrial Engineering, Purdue University, West Lafayette, IN; ${ }^{3}$ School of Agricultural \& Biological Engineering, Purdue University, West Lafayette, IN)

Journal: Human Factors and Ergonomics in Manufacturing \& Service Industries, 2012, 22 (3), p. 256268.

Objetivo(s): Testar a validação e confiabilidade da metodologia VID (Design virtual interativo) para avaliações ergonômicas e identificar os possíveis fatores que podem afetar sua validação e confiabilidade.

Os autores iniciam o artigo discutindo sobre a ergonomia voltada ao ambiente de trabalho, suas demandas e conseqüências, e logo e seguida tratam de modelos humanos digitais (DHM) para a avaliação ergonômica em locais de trabalho. Discutem os métodos de avaliação ergonômica que utilizam modelos físicos e virtuais, e afirmam que os métodos atuais para geração de movimentos humanos ainda apresentam muitos problemas. Fazem um breve referencial teórico sobre as tecnologias associadas ao VID (Design virtual interativo) - DHM, MOCAP e VE. Logo em seguida, descrevem a metodologia, os objetivos, ambiente, funcionamento e a integração entre as tecnologias utilizadas, possíveis erros e as hipóteses a serem testadas. Sobre a metodologia da pesquisa, foi criado um ambiente experimental no Centro avançado de Sistemas de Veículos na Universidade do Estado do Mississipi. São descritos todos os equipamentos utilizados, calibração do sistema e os procedimentos adotados. A simulação ocorreu em 3 níveis, a partir das hipóteses formuladas. 0 experimento foi realizado com 36 pessoas de ambos os sexos, convidados a partir de uma triagem. 0 artigo descreve mais detalhes sobre idade e altura das pessoas. Para a realização dos testes foram classificadas algumas tarefas. Foram feitos dois ensaios ergonômicos de cada tarefa, sendo as posturas assumidas registradas e analisadas. A respeito dos resultados, em termos gerais, o VID se mostrou uma metodologia que proporciona um ambiente de confiança para todas as tarefas de levantamento analisadas. A validação do VID foi demonstrada, contudo apenas em certas condições.

\section{- Artigo 5 -Using Virtual Reality to Examine Hazard Perception in Package Design.}

Autores: Hande Ayanoğlu ${ }^{1,2}$, Francisco Rebelo ${ }^{1,3}$, Emília Duarte ${ }^{1,4}$, Paulo Noriega ${ }^{1,3}$ e Luís Teixeira ${ }^{1,3}$ $\left({ }^{1}\right.$ Ergonomics Laboratory, Faculdade de Motricidade Humana, UTL, Portugal; ${ }^{2}$ IDEAS, Dipartimento di Industrial Design, Ambiente e Storia, Seconda Universitàdegli Studi di Napoli, Aversa (CE), Italy. ${ }^{3}$ CIPER - Interdisciplinary Center for the Study of Human Performance, UTL, Portugal; 4 Unidcom, IADE, Creative University, Lisboa, Portugal)

Congresso: $\mathrm{HCl}$ 2013. Design, User Experience, and Usability - User Experience in Novel Technological Environments. V. 11, Parte III, p. 30-39.

Objetivo(s): Apresentar os resultados de um estudo piloto sobre a eficácia do uso de uma metodologia baseada em RV para examinar a influência das características de embalagens (forma) na percepção de periculosidade dos usuários.

Os autores iniciam o artigo ressaltando a escassez de pesquisas no campo da ergonomia para embalagens. Foram objetos deste estudo - embalagens de líquidos perigosos domésticos, e de como os usuários são levados a perceber o risco pelo design da embalagem (forma). Os autores apresentam um experimento com 10 pessoas (na idade de 18 a 24 anos) em Portugal, utilizando a RV para o exame de percepção de risco no design de algumas embalagens. O experimento utiliza o ambiente virtual para visualizar os protótipos 3D das embalagens, obedecendo aos 
seguintes critérios: 1 . facilidade de discernir os detalhes dos protótipos 3D; 2 . os participantes deveriam ser capazes de observar facilmente as embalagens, bem como a navegar livremente no ambiente virtual (VE); O VE deveria ser apresentado em estereoscópica, a fim de proporcionar ao participante a informação da profundidade; 4. dar ao participante a possibilidade de navegar e mudar seu ponto de vista no VE; 5. a tarefa que os participantes precisariam cumprir dentro do VE não deveria ser tão complexa que os impedisse de responder verbalmente às perguntas enquanto eles estão interagissem com o VE. Foram utilizados como ferramentas: projetor de vídeo Lightspeed DepthQ 3D, Óculos 3D APG6000 da MacNaughtonm, um mouse e questionário. Os protótipos virtuais foram projetados usando Rhinoceros e depois exportados para o Unity. Principais resultados: o uso de protótipos em 3D em RV pode aumentar a percepção das embalagens devido a vários fatores, tais como: informações, visão estereoscópica, a perspectiva, navegação no ambiente e presença. Ao contrário de estudos que usam desenhos 2D que não demonstram os detalhes do produto, fazendo com que os usuários possam ter uma impressão errada sobre a forma correta das embalagens. Os resultados obtidos neste estudo piloto indicam que os participantes foram capazes de fazer um julgamento perceptivo da periculosidade das embalagens em 3D, bem como para avaliar o seu nível de familiaridade. Os resultados obtidos sugerem que a RV pode ser usada com sucesso para avaliar a percepção dos usuários de embalagens de conteúdos perigosos. Nas conclusões os autores consideram os estudos com RV e percepção de risco em embalagens, promissores e recomendam novas investigações em relação à percepção de risco, com amostras maiores e questionário mais detalhado, assim como examinar outras características das embalagens além de forma (como por exemplo: cor, textura, material).

\section{- Artigo 6 - Application of Virtual Reality Technologies in Consumer Product Usability.}

Autores: Christiane Soares Falcão; Marcelo Márcio Soares (PPGDesign UFPE, Recife, Brasil)

Congresso: $\mathrm{HCl}$ 2013. Design, User Experience, and Usability - User Experience in Novel Technological Environments. V. 12.

Objetivo(s): Apresentar uma abordagem conceitual sobre aplicações de tecnologias de realidade virtual na avaliação de usabilidade no design de produtos de consumo

Os autores iniciam o artigo conceituando a Realidade Virtual (RV), bem como suas possibilidades de uso e modalidades. Em seguida apresentam a contribuição da RV para design de produto, podendo ocorrer em diversas etapas do seu desenvolvimento. Os autores indicam que é na fase de concepção, principalmente na fase de prototipagem, o momento de maior benefício da sua aplicação. Através da aplicação de testes de usabilidade da interface do produto com simulações de uso através de protótipos digitais. Apresentam como vantagens do uso de modelos digitais tridimensionais em ambientes imersivos, o fato dos produtos poderem ser manipulados diretamente pelos usuários, permitindo feedback imediato, ainda na fase de desenvolvimento do produto; outra vantagem refere-se à construção virtual; além disso os cenários típicos dos usuários podem ser facilmente simulados em RV, como ambientes domésticos ou de escritórios; outra vantagem indicada é que, a solução e projeto podem ser conduzidos de forma interativa, mais barata, rápida e fácil sem a necessidade de construção de modelos físicos, aumentando assim, as possibilidades de alternativas ao serem desenvolvidas. Em síntese a RV permite soluções alternativas 
diferentes para o produto de consumo ser avaliado e comparado de forma bastante realista e dinâmica, nos diversos aspectos de interação - visual, sonoro e de forças. Em relação à visualização estéreoscópica e feedback háptico, a simulação em RV oferece uma interação mais realista com os protótipos de CAD (Computer Aided Design). Em relação ao modelo físico, os autores não descartam seu uso, e colocam que a RV, assim como os métodos físicos, não devem ser vistos como competitivos e sim complementares. Em seguida, os autores apresentam definições de usabilidade e os tipos de testes. O artigo finaliza, ressaltando a importância e necessidade de mais pesquisas sobre o impacto do desenvolvimento de sistemas de RV em testes de usabilidade de produtos.

\section{CONCLUSÃO}

Foram encontrados diversos trabalhos relativos ao uso de ferramentas virtuais no desenvolvimento de produtos e nas interações homem-produto ou homemambiente, em suas diversas etapas. Porém, a partir do foco de investigação - o uso de ferramentas de RV para a avaliação ergonômica/usabilidade de embalagens, ou ainda em produtos de consumo com características de uso semelhantes, foram encontradas poucas publicações, dentro do recorte temporal escolhido.

Pelo levantamento realizado, é possível perceber o potencial de uso de ambientes virtuais tanto no desenvolvimento e avaliação de produtos, envolvendo o usuário nas fases iniciais do projeto, quanto no projeto e avaliação de ambientes de trabalho. Em termos gerais, os autores apresentam como promissora a aplicação de RV nessas situações, principalmente pelo crescente acesso às tecnologias envolvidas.

Sobre as dificuldades enfrentadas pelas pessoas nos ambientes virtuais, os autores dos artigos selecionados, são quase que unânimes em apresentar uma preocupação com a interação dos usuários com os componentes técnicos de RV, no que diz respeito à usabilidade dos sistemas. Requerendo assim, cada vez mais, a realização de estudos mais aprofundados sobre a compatibilidade, conforto e segurança dos usuários quando inseridos no ambiente virtual. O que pode representar uma importante área de pesquisa para a Ergonomia.

No que se refere à pesquisas desenvolvidas, apesar do avanço existente do uso da RV no desenvolvimento e avaliação de produtos, desde sua implementação até os dias de hoje, este ainda é um campo bastante vasto e de grande potencial de investigações científicas. Principalmente no que se refere a sua aplicação em avaliações ergonômicas e de usabilidade de produtos. Observa-se ainda que, as aplicações dos sistemas de RV ainda ocorrem em maior número nas grandes corporações, principalmente nos setores da aviação e automobilismo.

Já para o número reduzido de pesquisas aplicadas a embalagens, algumas hipóteses podem ser formuladas: baixa disseminação da tecnologia em produtos de consumo de massa, pela "simplicidade" de uso, pelo custo reduzido do produto final em detrimento à outros tipos de produtos, dentre outras. O que demanda mais investigações a fim de conhecer as reais razões desse quadro. No caso de aplicações de RA em embalagens, já são encontradas mais estudos.

A partir desta pesquisa foi possível também compreender melhor as informações sobre os periódicos internacionais; conhecer melhor as ferramentas de busca; identificar autores, grupos e as pesquisas que estão desenvolvidas em várias 
partes do mundo, e, sobretudo obter informações para compor o estado da arte e as referências bibliográficas da tese em questão. Contudo, o levantamento realizado não teve o intuito de esgotar as possibilidades de pesquisa, ou seja, os periódicos e artigos pesquisados não configuram a totalidade dentro da temática tratada. Principalmente pelo fato da área de Ergonomia e usabilidade perpassar por diversas áreas de conhecimento.

\section{REFERÊNCIAS}

AKAGUI, Daniela; KIRNER, Cláudio. Desenvolvimento de Aplicações de Realidade Aumentada com ARToolKit. In: WRA - Workshop de Realidade Aumentada. Anais ... 2004. Piracicaba, SP. P. 6-8.

CAPES. Portal de Periódicos. Disponível em:http://www.periodicos. capes.gov.br. Acesso em 2013.

FALCÃO, Christiane F., SOARES, Marcelo. M. Ergonomics, Usability and virtual reality: a review applied to consumer product. Advances in Usability Evaluation. Part II. Edited by Rebelo, F.; Soares, M. M. USA: CRC Press - Taylor \& Francis Group. pp.297-306. 2012.

GRAVE, L. et al. A realidade virtual como ferramenta de treino pra montagem de cablagens elétricas. In: 10 Encontro Português de Computação Gráfica. 2001. Ed. J. A. Jorge. 147-63. Lisboa: INESC. Disponível em: http://virtual.inesc.pt/virtual/10epcg/acatas/pdfs/grave.pedf.

HCI INTERNATIONAL 2013. Anais. Springer. Las Vegas, NV, USA.

KIRNER, Cláudio. Evolução da Realidade Virtual no Brasil. In: Symposium of Virtual and Augmented Reality. João Pessoa, PB, SBC, p. 1-11. 2008. P. 1-11.

KIRNER, Claudio; KIRNER, Tereza Gonçalves. Evolução e Tendências da Realidade Virtual e da Realidade Aumentada. In: Realidade Virtual e Aumentada: Aplicações e Tendências. XIII Simpósio de Realidade Virtual e Aumentada. Sociedade Brasileira de Computação. 2011.

PRO TESTE - Associação Brasileira de Defesa do Consumidor; AMB - Associação Médica Brasileira (AMB). Acidentes de Consumo. (Cartilha). 2005.

REBELO, Francisco et al. Virtual Reality in Consumer Product Design: Methods and Applications.In: Edited by W. Karwoski; M. M. Soares; N. A. Staton. Human Factors and Ergonomics in Consumer Product Design: Methods and Techniques. USA: CRC Press - Taylor \& Francis Group. 2011. p. 381-402.

SILVA, Fernando et al. Sistema flexível de realidade aumentada de baixo custo com base em projecção estereoscópica. In Encontro Português de Computação Gráfica. Anais... 17, Covilhã, 2009.

TORI, R.; KIRNER, C. Fundamentos de Realidade Virtual. In: Romero tori; Claudio Kirner; Robson Siscouto. (Org.). Fundamentos e Tecnologia de Realidade Virtual e Aumentada. Porto Alegre: Sociedade Brasileira de Computação - SBC, 2006, v. 1, p. 7-22.

WILSON, John R. Virtual environment applications and applied ergonomics. Applied Ergonomics, v. 30, 1999, p. 3-9.

ZORZAL, Ezequiel Roberto; NUNES, Fátima de Lourdes dos Santos. Realidade Aumentada e suas Aplicações na Área de Saúde. In: Tendências e Técnicas em Realidade Virtual e Aumentada. Salvador: Sociedade Brasileira de Computação - SBC, 2014. v. 4, p. 49-62.

ZUNJIC, A. Ergonomics of Packaging. In: Edited by KARWOWSKI, Waldemar; SOARES, Marcelo Márcio. STANTON, Neville A. Human Factors and Ergonomics in Consumer Product Design: Methods and Techniques. USA: CRC Press - Taylor \& Francis Group. 2011. p.101-123. 